\title{
Development of non-invasive method for assessment of hepatic steatosis
}

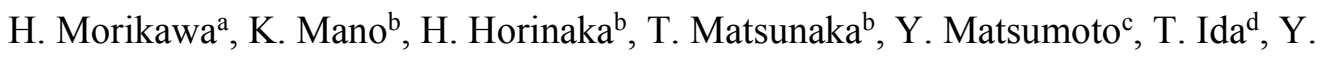

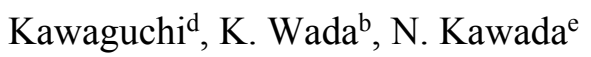 \\ ${ }^{a}$ Department of Premier Preventive Medicine, Graduate School of Medicine, Osaka \\ City University, Osaka, Japan. \\ ${ }^{\mathrm{b}}$ Department of Physics and Electronics, Graduate School of Engineering, Osaka \\ Prefecture University, Sakai, Japan. \\ ${ }^{\mathrm{c}}$ Graduate School of Human Life Science, Osaka City University, Osaka, Japan. \\ d ADVANTEST CORPOLATION, Tokyo, Japan. \\ e Department of Hepatology, Graduate School of Medicine, Osaka City University, \\ Osaka, Japan.
}

\begin{abstract}
Steatosis is a critical feature of liver disease and is considered to play a pivotal role in the progression of nonalcoholic fatty liver disease, as well as being a surrogate marker of metabolic syndrome. The purpose of this study was to develop a non-invasive diagnostic method for assessment of liver steatosis. It is well known that ultrasonic velocity depends on materials and temperature. For example, the ultrasonic velocity in water is $1530 \mathrm{~m} / \mathrm{s}$ at $37^{\circ} \mathrm{C}$ and $1534 \mathrm{~m} / \mathrm{s}$ at $39^{\circ} \mathrm{C}$, while that in fat is $1412 \mathrm{~m} / \mathrm{s}$ at $37^{\circ} \mathrm{C}$ and $1402 \mathrm{~m} / \mathrm{s}$ at $39^{\circ} \mathrm{C}$. On this basis, we thought that the percentage of fat in hepatic steatosis could be assessed by detecting changes of ultrasonic in the liver, caused by
\end{abstract}


warming. In order to confirm the effectiveness of this method, we obtained the ultrasonic velocity changes of tissue phantom including lard oil and the liver of living rabbit by ultrasonic warming, and then succeeded in 2-D imaging of ultrasonic velocity changes of the phantom and the liver of living rabbit. We named this the ultrasonic velocity-change method. The experimental results show the possibility that hepatic steatosis could be characterized using our novel, non-invasive method.

Key words: ultrasonic velocity change, hepatic steatosis, pulse shift, temperature

\section{Introduction}

Liver steatosis results from the accumulation of fatty droplets in the hepatocytes. There are several causes, such as alcohol consumption, viral hepatitis and metabolic dysfunction. Liver steatosis is a reversible, benign condition and a common abnormality occurring in approximately $30 \%$ of the general population in the United States [1]. However, in many cases, steatosis can be associated with inflammation (i.e. steatohepatitis), which may result in liver fibrosis and can progress to cirrhosis, liver failure and hepatocellular carcinoma [2]. Recently, hepatocellular carcinoma also has been recognized as a complication of liver steatosis and emerging evidence suggests that cardiovascular disease may also be more common in patients with steatosis, even when adjusted for traditional risk factors [3, 4]. Liver biopsy is regarded as the gold standard for the assessment of steatosis. However, its use in clinical practice is limited because of its invasiveness. Therefore, various imaging methods, including ultrasonography (US), computed tomography (CT), magnetic resonance imaging (MRI) and proton magnetic

resonance spectroscopy (MRS), have been used for the non-invasive evaluation of 
steatosis. US commonly detects changes in the liver parenchyma as increased echogenicity compared to the kidney and is the most frequently used tool in clinical practice. The sensitivity of US ranges from $60 \%$ to $94 \%$, with a specificity between $88 \%$ and $95 \%$ [5-7]. The major drawbacks of ultrasonography are operator dependency and are affected considerably by increasing body mass. The sensitivity improves to $80 \%$ when liver fat exceeds $30 \%$, but drops to $50 \%$ in morbid obesity or when the liver fat content is below 20\% [8-10]. CT provides a better estimation of liver fat. Estimation of liver fat content is based on the comparison of hepatic and splenic attenuations and can predict moderate to severe degrees of steatosis with greater sensitivity when steatosis is above $33 \%$ of the liver parenchyma [11]. Both US and CT are considered qualitative tests, best suited to detect liver steatosis but not to quantify the amount of fat or to be used to follow disease progression or patients' responses to treatment. On the other hand, MRS has a very good correlation with the amount of liver fat estimated by liver biopsy [12]. The lower limit of MRS is considered to be $5 \%$ of the liver wet weight as hepatic triglycerides content [13]. MRS usually can be performed within 20 to 30 minutes but is currently limited to academic centers.

The purpose of this study was to develop a non invasive and inexpensive diagnostic method for assessment of liver steatosis. It is well known that the ultrasonic velocities of various materials have inherent values and vary with temperature. For example, the ultrasonic velocity in water is $1530 \mathrm{~m} / \mathrm{s}$ at $37^{\circ} \mathrm{C}$ and $1534 \mathrm{~m} / \mathrm{s}$ at $39^{\circ} \mathrm{C}$, while that in fat is $1412 \mathrm{~m} / \mathrm{s}$ at $37^{\circ} \mathrm{C}$ and $1402 \mathrm{~m} / \mathrm{s}$ at $39^{\circ} \mathrm{C}$. The rate of change of the ultrasonic velocity in water is $+2 \mathrm{~m} / \mathrm{s}$ at $37^{\circ} \mathrm{C}$ and that in fat is $-4 \mathrm{~m} / \mathrm{s}$ at the same temperature $[14,15]$. The ultrasonic velocity increases in muscle and internal organs with a high percentage of water content, but decreases in fatty tissue. On this basis, we thought that the fat in 
hepatic steatosis could be assessed by detecting ultrasonic velocity change of the liver caused by warming. In order to confirm the effectiveness of this method, we obtained the ultrasonic velocity changes of tissue phantom including lard oil and the liver of living rabbit by ultrasonic warming, and then succeeded in 2-D imaging of ultrasonic velocity changes of the phantom and the living rabbit liver. We named this the method ultrasonic velocity-change method. We wished to confirm whether this method can provide a quantitative evaluation when the liver fat content is between $5 \%$ and $20 \%$. The experimental results showed the possibility that the assessment of hepatic steatosis could be achieved using our novel and noninvasive imaging method.

\section{Principle of ultrasonic velocity-change method}

In general, biological tissues consist mainly of water and their ultrasonic velocities increase with rising temperature. On the other hand, the velocities of tissues containing fat decrease with rising temperature. The ratio of ultrasonic velocity change is $+1.9 \mathrm{~m} / \mathrm{s}$ in water but it is $-4.9 \mathrm{~m} / \mathrm{s}$ in fat, therefore, it may be possible to quantify the percentage of fat on the basis of the change in ultrasonic velocity with temperature. In the liver, the change in ultrasonic velocity with respect to temperature depends heavily on the percentage of fat [16]. Fig.1 shows the basic experimental setup for the ultrasonic velocity-change method, which consists of a water tank kept at constant temperature, a block containing fat dispersed in water, the ultrasonic linear array transducer and the warming ultrasonic transducer. The ultrasonic linear array transducer transmits and receives the ultrasonic pulse wave. The ultrasonic pulses emitted from the array transducer are reflected from the various boundaries of different acoustic impedance. As shown in Fig.1, the echo pulses reflected at the boundaries shift according to the 
ultrasonic velocity change caused by the local temperature rise $[17,18]$ with the warming ultrasonic transducer.

The echo pulses reflected at the boundary surfaces I and II are received by the same transducer. After warming, the echo pulse becomes faster when it propagates in the water-rich domain and reflects back from boundary surface I $\left(\Delta \tau_{1}\right)$, but becomes slower when it propagates in the fat containing domain and reflects back from boundary surface II $\left(\Delta \tau_{2}\right)$. Consequently, the difference in shift may be expressed as $\Delta \tau_{1}+\Delta \tau_{2}$. The pulse shift between the bi-border $\left(\Delta \tau_{\mathrm{n}}\right)$ distinguishes the latter shift $\left(\Delta \tau_{\mathrm{n}-1}+\Delta \tau_{\mathrm{n}}\right)$ from the former $\left(\Delta \tau_{\mathrm{n}-1}\right)$. The round trip time of the echo pulse between the boundaries and the time difference are denoted by $\tau_{\mathrm{n}}$ and $\Delta \tau_{\mathrm{n}}$, respectively, so that the velocity change, $\Delta \mathrm{V}_{\mathrm{n}}$, of the warmed region may be represented by $\Delta v_{n}=v \frac{\Delta \tau_{n}}{\tau_{n}}$, where $\mathrm{v}$ is the ultrasonic velocity.

The echo waveform of each acoustic scan line was divided into appropriate sections with the width of the transmitted pulse. The cross-correlation between the corresponding section of the waveform data stored before and after warming was calculated to obtain the time difference, $\Delta \tau$, of the echo pulse shift induced by the temperature change. The ultrasonic velocity-change is constructed from $\Delta \tau$ and $\tau$ for each acoustic scan line.

The ultrasonic velocity change $\Delta v$ by the temperature change $\Delta T$ is represented as follows,

$$
\frac{\Delta v}{\Delta T}=\left(\frac{\Delta v}{\Delta T}\right)_{w} \cdot x+\left(\frac{\Delta v}{\Delta T}\right)_{f}(1-x)
$$


where $x$ is the fat content, $(\Delta \mathrm{v} / \Delta \mathrm{T})_{w}$ is the temperature dependence of ultrasonic velocity in water and $(\Delta \mathrm{v} / \Delta \mathrm{T})_{f}$ is that in fat. As $(\Delta \mathrm{v} / \Delta \mathrm{T}) \mathrm{W}$ and $(\Delta \mathrm{v} / \Delta \mathrm{T}) \mathrm{f}$ can be determined in advance, the fat percentage $\mathrm{x}$ can be determined from the $\Delta \mathrm{v}$ and $\Delta \mathrm{T}$ values of the object. $\Delta \mathrm{v}$ can be obtained by the ultrasonic velocity-change imaging method. $\Delta \mathrm{T}$ is required for the calculation.

\section{Experimental verification for effectiveness of the imaging method}

\subsection{Preliminary experiment using tissue phantom}

Before the experiment using living rabbits, we assessed different fat content of the tissue phantoms containing lard oil manufactured by OST Co., Ltd. (Tokyo, Japan). The lard-free phantom was composed of water, agar, talc, thickener and 1- propanol, and the proportions (by weight) of the water, agar, talc, thickener and 1- propanol were 85.54, $1.20,3.60,1.20$ and, $8.46 \%$, respectively. The lard phantom manufactured by adding sodium dodecyl sulfate and lard to the lard-free phantom. A conventional pulse echo system (5077PR, Olympus) was used to obtain the pulse shift in the lard-free phantom and the phantoms containing $10 \%, 20 \%$ and $30 \%$ lard. We measured the ultrasonic velocity in each phantom at $30^{\circ} \mathrm{C}$. Then, we obtained the ultrasonic velocity change for each phantom at various temperatures between $30^{\circ} \mathrm{C}$ and $40^{\circ} \mathrm{C}$. Fig. 2 shows that the ultrasonic velocity changes depend on the percentage of fat. Linear approximations of the velocity change rates of the lard-free phantom and the phantoms containing $10 \%$, $20 \%$ and $30 \%$ lard gave values of $0.090,-0.001,-0.110$ and -0.183 , respectively. This shows that the fat contents of the phantoms were assessed quantitatively by ultrasonic velocity change under the given temperature change.

\subsection{Experiment using living animal}


Figure 3 shows the experimental system configuration which consists of the ultrasonic imaging equipment (Model:SSD-500,Aloka, Japan) with the $7.5 \mathrm{MHz}$ linear array probe assembled with 128 channels, the high speed signal processing unit (PX-I, National Instruments Corporation, Austin, TX) that converts continuous RF signals of SSD-500 to digital signals at $10 \mathrm{bits}$ and $100 \mathrm{MHz}$ and records continuously these signals in the storage module, and the warming circular ultrasonic transducer $(35 \mathrm{~mm}$ in diameter, US710, Ito Co., Ltd, Tokyo, Japan) driven by a continuous sine wave of $1 \mathrm{MHz}$. The tip of the warming ultrasonic transducer has an attachment of silicone resin for good contact with the skin surface. The output of the warming transducer is $1 \mathrm{~W} / \mathrm{cm}^{2}$ and within the safety level of living tissue. We developed software for correction of the movement by breathing and heartbeat during acquisition of velocity change data.

Pathogen-free male Japanese White rabbits, about 1-year-old and weighing 3.0 to 3.5 $\mathrm{kg}$, were obtained from Oriental Yeast (Tokyo, Japan). The rabbits were housed at a constant temperature, divided into two groups and given either $100 \mathrm{~g} / \mathrm{day}$ of a standard $\operatorname{diet}(\mathrm{SD})$ (n=3, CR3 obtained from CLEA Japan Inc., Tokyo, Japan) or a high-fat and cholesterol diet (HFD) $(n=6)$, which consisted of SD supplemented with $12 \%$ corn oil and $1.50 \%(\mathrm{w} / \mathrm{w})$ cholesterol, for $2-4$ weeks $[19,20]$. The rabbits were fasted for 24 hours before ultrasonography and sacrifice. Then, they were given an intravenous anesthetic, examined and laparotomized for blood and liver sampling. For removal of the liver, the portal vein of each animal was cannulated using an 18-gauge Teflon catheter and the liver was perfused with $100 \mathrm{ml}$ of PBS to remove the blood. After harvesting, the liver was cut into pieces and the samples were either frozen or fixed in 4\% paraformaldehyde. The experiments were conducted humanely in accordance with 
the recommendations of the Guide for the Care and Use of Laboratory Animals of Osaka City University School of Medicine.

The ultrasonic velocity change in each phantom was measured in a constant temperature bath which permitted temperature variation from $32^{\circ} \mathrm{C}$ to $42^{\circ} \mathrm{C}$.In the animal model, we performed warming for 60 seconds and stored the moving digital images for 10 seconds after switching off the warming source.

Paraformaldehyde-fixed specimens were cut into $5 \mu \mathrm{m}$ thick sections and stained with H\&E. Frozen sections ( $5 \mu \mathrm{m}$ thick) were stained with Oil red O (Wako Pure Chemical Co., Osaka, Japan). The areas stained by Oil red $\mathrm{O}$ were measured using an image analyzer (Lumina Vision, Mitani Corporation, Tokyo, Japan) to quantify the areas of lipid deposition.

Serum levels of aspartate aminotransferase (AST) and alanine aminotransferase (ALT) were measured at the Special Reference Laboratories (Osaka, Japan). As biochemical parameters, levels of total cholesterol (TC), triglycerides (TG), and fatty acids (FA) in the livers were measured by Skylight Biotech (Akita, Japan) using the Folch method. Analyses were performed using JMP-11 software (SAS Institute, Cary, NC). Linear regression analysis was performed between the change rate of the ultrasonic velocity and quantitative histologic analyses for fat accumulation and lipid extraction in each rabbit.

As shown in Table 1, the liver weights of the rabbits on the HFD were significantly higher than those of rabbits on the SD (Fig.4). More hepatocytes were positive for Oil red O staining in the HFD group than in the SD group (Fig.5). Furthermore, the hepatic TC content was significantly higher in the HFD group (HFD2w, 39.2 $\pm 3.4 \mathrm{mg} / \mathrm{g}$; HFD4W, $50.9 \pm 15.3 \mathrm{mg} / \mathrm{g})$ than the SD group $(2.7 \pm 0.5 \mathrm{mg} / \mathrm{g})$, although the hepatic 
TG content was similar. These data indicate that, in this animal model, the lipid droplets in the hepatocytes are composed mostly of cholesterol. Simple regression analyses of the relationship between histology and biochemical parameters indicated that the area positive for Oil red $\mathrm{O}$ staining correlated significantly with the hepatic lipid, made up of TC, TG and free cholesterol $(\mathrm{r}=0.808, \mathrm{p}<0.01)$.

We assessed the performance of the ultrasonic velocity-change method in vivo using the living rabbit model. The echo data of 100 lines in the central portion of the 256 lines constituting the B-mode image were employed for determination of the ultrasonic velocity change. The ultrasonic velocity change was calculated from the echo waveforms on each line. The average and the standard dispersion of values of ultrasonic velocity change values of 100 lines were calculated. Fig. 6 shows a high correlation between the rate of change of ultrasonic velocity and the histochemical quantification of lipid droplets $(\mathrm{r}=0.80, \mathrm{p}<0.001)$.

\section{Discussion}

In this study, the novel principle of ultrasonic velocity change dependent on temperature was implemented successfully in an animal model of steatosis. If this system can be developed for clinical examination, it should provide a non-invasive, inexpensive and rapid method for detection of fat in the human liver. This study is the first to report the development of a new method for quantification of fat.

Although Horinaka et al. studied the first system using optical assistance for warming $[17,18]$, we used low frequency ultrasound for warming deeper tissue. The safety of the transducer used for warming has been confirmed and the ultrasonic transducer (1or2MHz) is suitable for warming the liver, which is a deep organ. We assume that 
stable warming is possible at a range of $10 \mathrm{~cm}$ from the surface of the human body. Regarding the timing of the measurement of the change of ultrasonic velocity, we assessed this before and after the warming process. When we accumulated the data for warming, we found that measurement during cooling was quite reliable. Therefore, we carried out the measurement from the maximum temperature until the normal temperature was achieved. In the animal experiments, the temperature change in the liver was minimal. When the rabbits' livers were warmed by the ultrasonic transducer, the organs received no damage according to the normal alanine aminotransferase (ALT) value and comparison with sham operation.

The Dixon method of MRI and MRS are widely regarded as the most accurate noninvasive methods for the clinical assessment of hepatic steatosis [21-23]. However, MRI techniques and MRS are somewhat impractical because of their lengthy scan times, their reliance on the compliance of the patient, respiratory artifacts and particular locality, and they typically sample only a portion of the liver. This new methodology based on ultrasonography has the advantage of being non-invasive, with a short scan time, no respiratory artifacts in human patients, occupying little space and assessing a significant proportion of the liver.

Animal models may be critical for the evaluation of novel methodologies and, in this study, harvesting of the entire livers from the rabbits enabled accurate assessment of fat accumulation. This is not possible in humans because only small biopsy samples may be available $[24,25]$. Although this new method was validated in the animal model by correlation with the post-mortem results, including histologic analysis and hepatic lipid extraction, additional clinical validation is required in patients, correlating the imaging results with examination of biopsy tissue. In addition, the reproducibility of this method 
must be determined before the clinical utility can be ascertained. Although investigators usually remove the animal liver for analysis, there may be individual variation, such that the model is not suitable for the calibration of an imaging system. We developed the phantom consisting of lard as a physical model of fat accumulation. This phantom was used for optimization and comparison in this system.

Our study has several limitations. A major limitation was the inability to measure the temperature directly and noninvasively in the rabbit and human livers. Although the change rate of ultrasonic velocity could be obtained without precise temperature data, the accurate measurement of the deep body temperature contributed to improving quantitation and safety. There are methods for estimating deep body temperature that rely on calculation of thermal diffusion in the bloodstream and the coefficient of heat conduction from the border between the liver and the kidney, which does not accumulate fat. Safety is an important consideration for future human application of this method in preclinical or clinical studies. According to the American Institute of Ultrasound in Medicine (AIUM), no significant adverse biological effects were observed due to temperature increases of less than or equal to $2^{\circ} \mathrm{C}$ above normal for exposure durations up to 50 hours. The experiments in vivo using the living rabbit model were made under $1 \mathrm{~W} / \mathrm{cm}^{2}$ of warming transducer. The maximum temperature increase in the livers of the rabbits is estimated to be $2.0^{\circ} \mathrm{C}$. The intensity of ultrasonic for warming can be set lower because the ultrasonic velocity change can be detected even under a temperature change of $0.5^{\circ} \mathrm{C}$. Another limitation is the influence of the respiration artifact in the animal model. We carried out the experiments with the rabbits under anesthesia and observed the respiration and heartbeat artifacts. In order to use this technique on the living body, the images must be constructed in the presence of 
respiration and heartbeat. In this evaluation of the ultrasonic velocity change method, we developed new software for image construction and this enabled clear images to be obtained from the anesthetized rabbits. The experimental results showed that the ultrasonic velocity change-images clearly displayed fat accumulation. The software is based on template matching using Zero-mean Normalized Cross Correlation [26]. However, image construction for humans will be simpler than the animal model because patients can hold their breath during measurement.

Subcutaneous fat did not affect the measurement in the rabbit experiment but, in patients, this produces individual differences in terms of the depth of measurement. Even though subcutaneous fat did not affect the image constructed from the pulse signal, it may affect the warming process. The ultrasonic wave can warm a wide area when the output of the wave form is changed but it can be focused on the measurement area for precision warming.

\section{Conclusion}

We have evaluated the ultrasonic velocity-change method as a new methodology and analyzed the strong correlation with conventional fat quantitation methods in a living rabbit model of non-alcoholic steatohepatitis. Our experimental results provide evidence that the ultrasonic velocity-change method enables accurate quantification of liver fat in the living rabbit and has potential for the quantitative assessment of hepatic steatosis in clinical practice.

\section{Acknowledgements}


This study was supported by research grants from the Cannon Foundation (2011-2012), JST-SENTAN program (2012-2014), and JSPS KAKENHI Grant Number 15575203. The authors acknowledge the technical contributions of Misaki Higashiyama, Ryota Akiyama and Yukihiro Nomura. 


\section{References}

[1] L.S. Szczepaniak, P. Nurenberg, D. Leonard, J.D. Browning, J.S. Reingold, S. Grundy, H.H. Hobbs, R.L. Dobbins, Magnetic resonance spectroscopy to measure hepatic triglyceride content: prevalence of hepatic steatosis in the general population, Am. J. Physiol. Endocrinol. Metab. 288 (2005) E462-E468.

[2] G.C. Farrell, Fatty liver disease: NASH and related disorders, Blackwell Publishing, Malden, MA, USA, 2004.

[3] G. Targher, L. Bertolini, F. Poli, S. Rodella, L. Scala, R. Tessari, L. Zenari, G. Falezza, Nonalcoholic fatty liver disease and risk of future cardiovascular events among type 2 diabetic patients, Diabetes 54 (2005) 3541-3546.

[4] G. Targher, L. Bertolini, R. Padovani, S. Rodella, R.Tessari, L. Zenari, C. Day, G. Arcaro, Prevalence of nonalcoholic fatty liver disease and its association with cardiovascular disease among type 2 diabetic patients, Diabetes Care 30 (2007) 12121218.

[5] Z.M. Younossi, T. Gramlich, C.A. Matteoni, N. Boparai, A.J. McCullough, Nonalcoholic fatty liver disease in patients with type 2 diabetes, Clin. Gastroenterol. Hepatol. 2 (2004) 262-265. 
[6] P. Gupte, D. Amarapurkar, S. Agal, R. Baijal, P. Kulshrestha, S. Pramanik, N. Patel, A. Madan, A. Amarapurkar, A. Hafeezunnisa, Non-alcoholic steatohepatitis in type 2 diabetes mellitus, J. Gastroenterol. Hepatol. 19 (2004) 854-858.

[7] M. Hamaguchi, T. Kojima, N. Takeda, T. Nakagawa, H. Taniguchi, K. Fujii, T. Omatsu, T. Nakajima, H. Sarui, M. Shimazaki, T. Kato, J. Okuda, K. Ida, The metabolic syndrome as a predictor of nonalcoholic fatty liver disease, Ann. Intern. Med. 143 (2005) 722-728.

[8] B. Palmentieri, I. de Sio, V. La Mura, M. Masarone, R. Vecchione, S. Bruno, R. Torella, M. Persico, The role of bright liver echo pattern on ultrasound B-mode examination in the diagnosis of liver steatosis, Dig. Liver Dis. 38 (2006) 485-489.

[9] L.A. Adams, A. Paul, Role of liver biopsy and serum markers of liver fibrosis in non-alcoholic fatty liver disease, Clin. Liver Dis. 11 (2007) 25-35.

[10] C.K. Ryan, L.A. Johnson, B.I. Germin, A. Marcos, One hundred consecutive hepatic biopsies in the workup of living donors for right lobe liver transplantation, Liver Transpl. 8 (2002) 1114-1122.

[11] S. Saadeh, Z.M. Younossi, E.M. Remer, T. Gramlich, J.P. Ong, M. Hurley, K.D. Mullen, J.N. Cooper, M.J. Sheridan, The utility of radiological imaging in nonalcoholic fatty liver disease, Gastroenterology 123 (2002) 745-750. 
[12] C. Thomsen, U. Becker, K. Winkler, P. Christoffersen, M. Jensen, O. Henriksen, Quantification of liver fat using magnetic resonance spectroscopy, Magn. Reson. Imaging 12 (1994) 487-495.

[13] R. Ali, K. Cusi, New diagnostic and treatment approaches in non-alcoholic fatty liver disease (NAFLD), Ann. Med. 41 (2009) 265-278.

[14] M. Greenspan, C. Tschiegg, F. Breckenridge, A Progressive - Wave Velocimeter and the Speed of Sound in Water, J. Acoust. Soc. Am. 29 (1957) 763.

[15] S.A. López-Haro, L. Leija, L. Favari, A. Vera, Measurement of Ultrasonic properties into biological tissues in the hyperthermia temperature range, Physics Procedia 3 (2010) 551-558.

[16] Bamber JC, Hill CR. Ultrasonic attenuation and propagation speed in mammalian tissue as a function of Temperature. Ultrasound Med. Biol. 1979; 5:149-158.

[17] H. Horinaka, T. Matsunaka, T. Iwade, T. Masumura, T. Kobayashi, T. Kiuchi, K. Wada, Y. Cho, Optical Imaging in Scattering Medium by Detection of Ultrasonic Phase Shift Due to Light Illumination, Jpn. J. Appl. Phys. 41 (2002) 3555-3558.

[18] H. Horinaka, T. Ura, Y. Nakatani, K. Wada, T. Matsunaka, Spectroscopic ThreeDimensional Imaging of Light Scattering Medium by Detection of Ultrasonic Velocity Change owing to Light Illumination, Jpn. J. Appl. Phys. 45 (2006) 4761-4763. 
[19] Ogawa T, Fujii H, Yoshizato K, Kawada N. A human-type nonalcoholic steatohepatitis model with advanced fibrosis in rabbits. Am J Pathol. 2010; 177:153165.

[20] Ghoshal, G., Lavarello, R.J., Kemmerer, J.P., Miller, R.J., and Oelze, M.L., Ex vivo study of quantitative ultrasound parameters in fatty rabbit livers. Ultrasound Med. Biol. 38:2238-2248, 2012.

[21] S. McPherson, J.R. Jonsson, G.J. Cowin, P. O'Rourke, A.D. Clouston, A. Volp, L. Horsfall, D. Jothimani, J. Fawcett, G.J. Galloway, M. Benson, E.E. Powell, Magnetic resonance imaging and spectroscopy accurately estimate the severity of steatosis provided the stage of fibrosis is considered, J. Hepatol. 51 (2009) 389-397.

[22] B.K. Kang, E.S. Yu, S.S. Lee, Y. Lee, N. Kim, C.B. Sirlin, E.Y. Cho, S.K. Yeom, J.H. Byun, S.H. Park, M.G. Lee, Hepatic fat quantification: a prospective comparison of magnetic resonance spectroscopy and analysis methods for chemical-shift gradient echo magnetic resonance imaging with histologic assessment as the reference standard, Invest. Radiol. 47 (2012) 368-375.

[23] F.H. Cassidy, T. Yokoo, L. Aganovic, R.F Hanna, M. Bydder, M.S. Middleton, G. Hamilton, A.D. Chavez, J.B. Schwimmer, C.B. Sirlin, Fatty liver disease: MR imaging techniques for the detection and quantification of liver steatosis, Radiographics 29 (2009) 231-260. 
[24] C.D. Hines, H. Yu, A. Shimakawa, C.A. McKenzie, T.F. Warner, J.H. Brittain, S.B. Reeder, Quantification of hepatic steatosis with 3-T MR imaging: validation in ob/ob mice, Radiology 254 (2010) 119-128.

[25] H.A. Marsman, J.R. van Werven, A.J. Nederveen, F.J. Ten Kate, M. Heger, J. Stoker, T.M. van Gulik, Noninvasive quantification of hepatic steatosis in rats using 3.0 T 1H-magnetic resonance spectroscopy, J. Magn. Reson. Imaging 32 (2010) 148-154.

[26] J. Ooi, K. Rao, New insights into correlation-based template matching, Proc. SPIE 1468, Applications of Artificial Intelligence IX, Orlando, FL, (1991) 740-751.

\section{Figure legends}

Figure 1. Principle of the ultrasonic velocity change imaging method Left, schema of the position of the ultrasonic transducers for imaging and heating. Right, the sequential of pulse shift.

I, II: tissue boundaries.

Figure 2. Measurement of ultrasonic velocity by the Pulser-Receiver in the physical model.

Figure 3. Application of the ultrasonic velocity change imaging system to an animal model. 
A. An image of the experiments with the rabbit under anesthesia. B. Schema of the system applied to the animal model.

Figure 4. Histochemical observation of hepatic steatosis by oil red $\mathrm{O}$ staining. SD, standard diet; HFD, high fat diet. The black bar is $200 \mu \mathrm{m}$.

Figure 5. Macroscopic image of hepatic steatosis in the removed liver. SD, standard diet; HFD, high fat diet.

Figure 6. Measurement of the rate of change of ultrasonic velocity in the animal model using our system. Open circles, standard diet; filled circles, high fat diet. 
Table 1. Characteristics of the rabbit model

\begin{tabular}{|c|c|c|c|c|c|c|}
\hline & $\mathrm{BW}[\mathrm{g}]$ & LW [g] & Area $[\%]$ & $\mathrm{TC}[\mathrm{mg} / \mathrm{g}]$ & $\mathrm{TG}[\mathrm{mg} / \mathrm{g}]$ & Lipid $[\mathrm{mg} / \mathrm{g}]$ \\
\hline SD-1 & 3900 & 68 & 1.16 & 2.8 & 19.5 & 24.9 \\
\hline SD-2 & 3100 & 60 & 0.68 & 2.2 & 12.3 & 16.9 \\
\hline SD-3 & 3400 & 77 & 2.83 & 3.1 & 37.3 & 42.9 \\
\hline HFD2W-1 & 3200 & 86 & 2.20 & 43.1 & 27.3 & 76.9 \\
\hline HFD2W-2 & 3200 & 99 & 8.87 & 36.8 & 19.7 & 63.1 \\
\hline HFD2W-3 & 3200 & 85 & 6.52 & 37.7 & 23.6 & 67.6 \\
\hline HFD4W-1 & 3300 & 104 & 4.69 & 33.7 & 13.9 & 56.1 \\
\hline HFD4W-2 & 3200 & 108 & 9.61 & 56.0 & 43.8 & 107.7 \\
\hline HFD4W-3 & 3300 & 102 & 8.37 & 63.1 & 21.3 & 93.4 \\
\hline $\begin{array}{l}\text { SD, standa } \\
\text { cholesterol }\end{array}$ & $\begin{array}{l}\text { liet; HFD } \\
\text {, triglyce }\end{array}$ & $\begin{array}{l}\text { high fat } \\
\text { des. }\end{array}$ & iet; $\mathrm{BW}, \mathrm{bc}$ & ody weight; & $\mathrm{LW}$, liver we & ight; TC, total \\
\hline \multicolumn{7}{|c|}{ Area, measurement of the positive area in Oil red O staining. } \\
\hline \multicolumn{7}{|c|}{ The values of TC, TG, and lipid are given for 1 gram of the liver. } \\
\hline
\end{tabular}


Ultrasonic array transducer

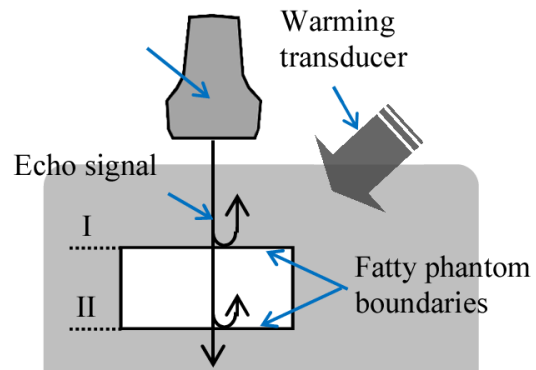

Tissue phantom

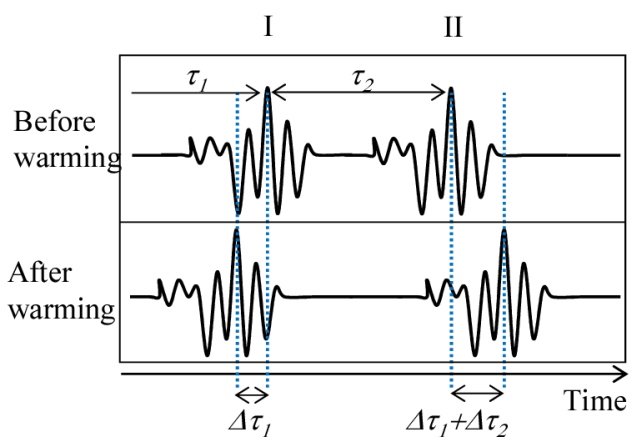




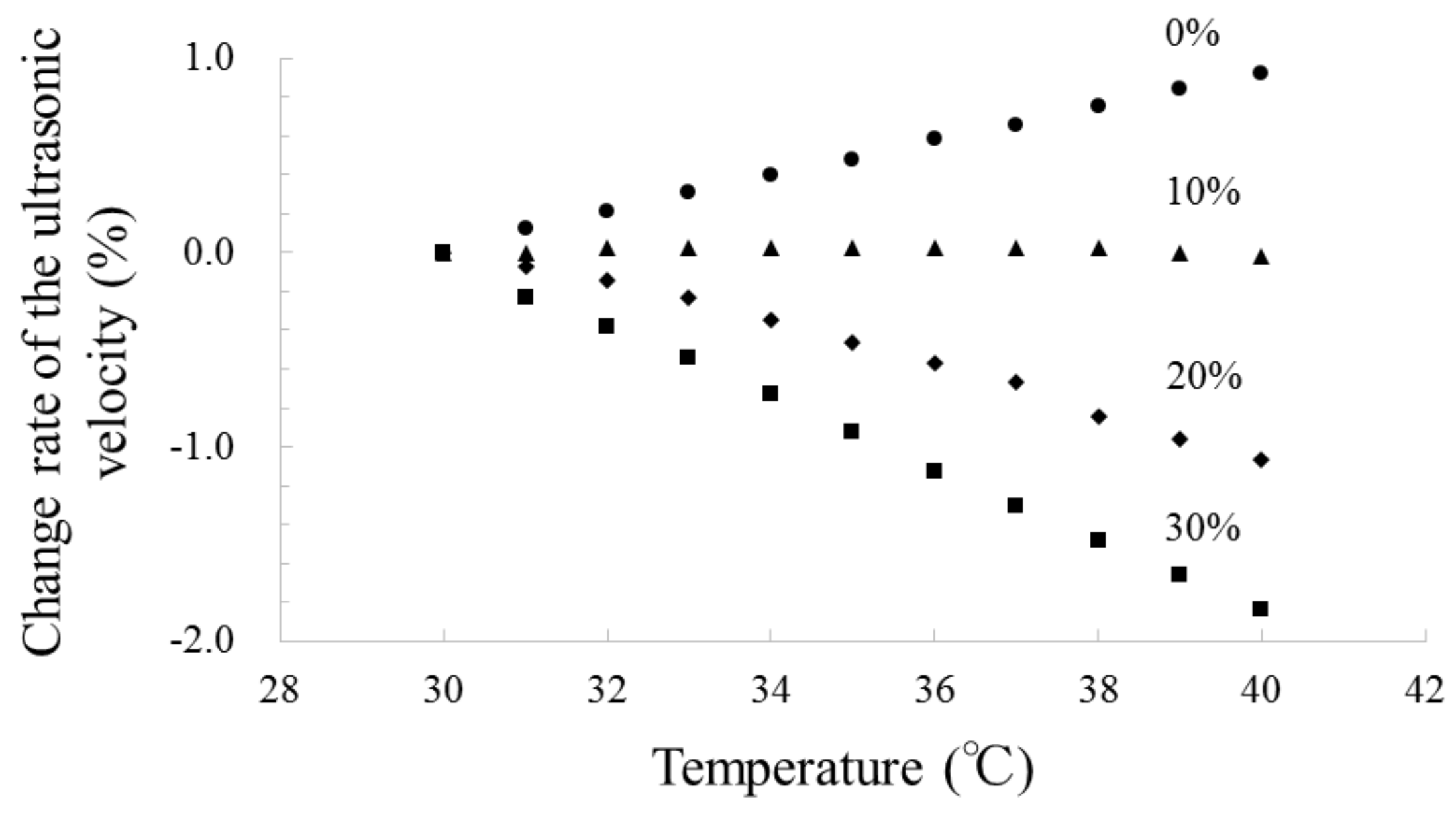


Figure 3

A

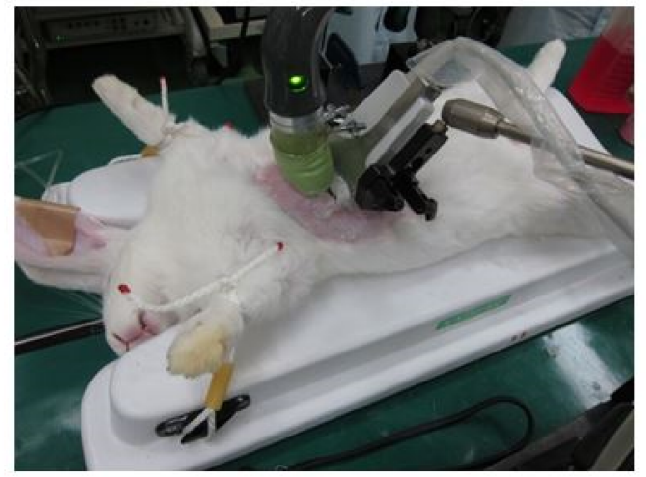

B

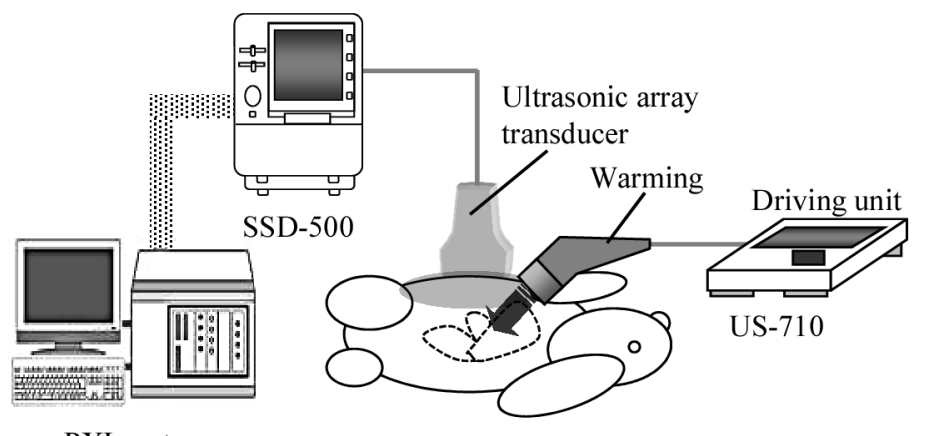

PXI-system 
Figure 4
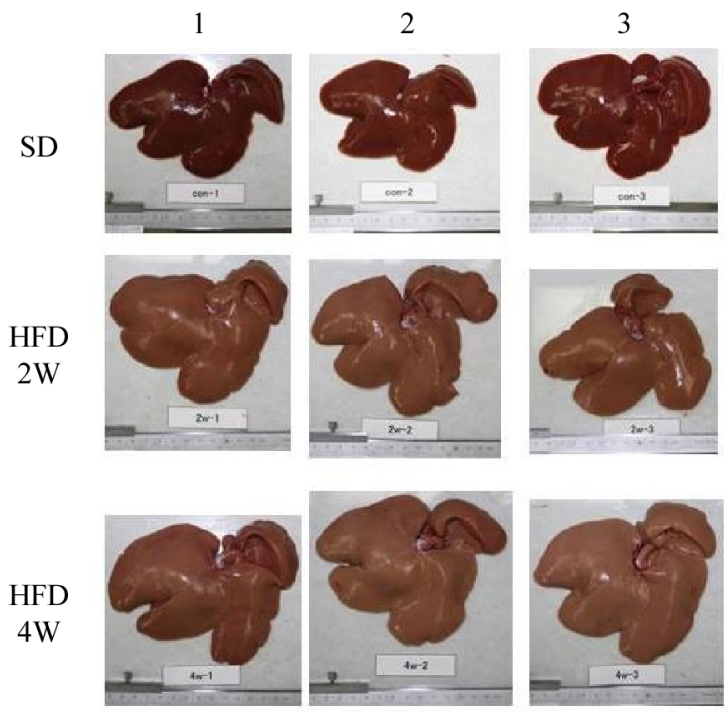


\section{Figure 5}

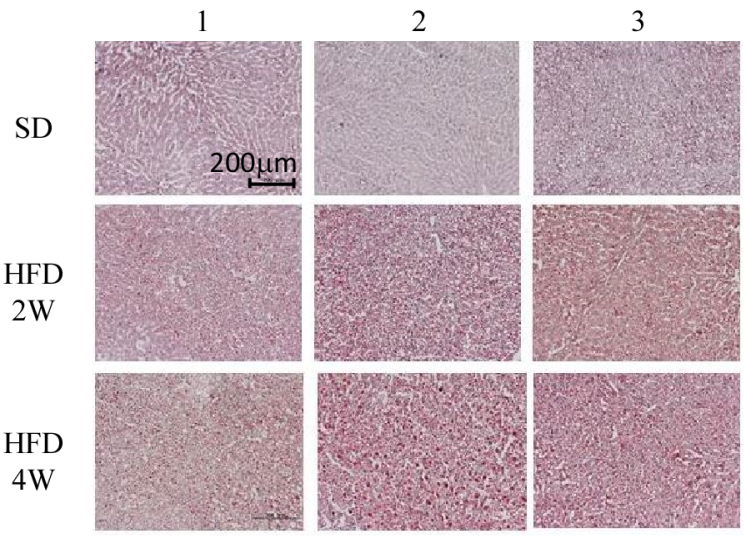




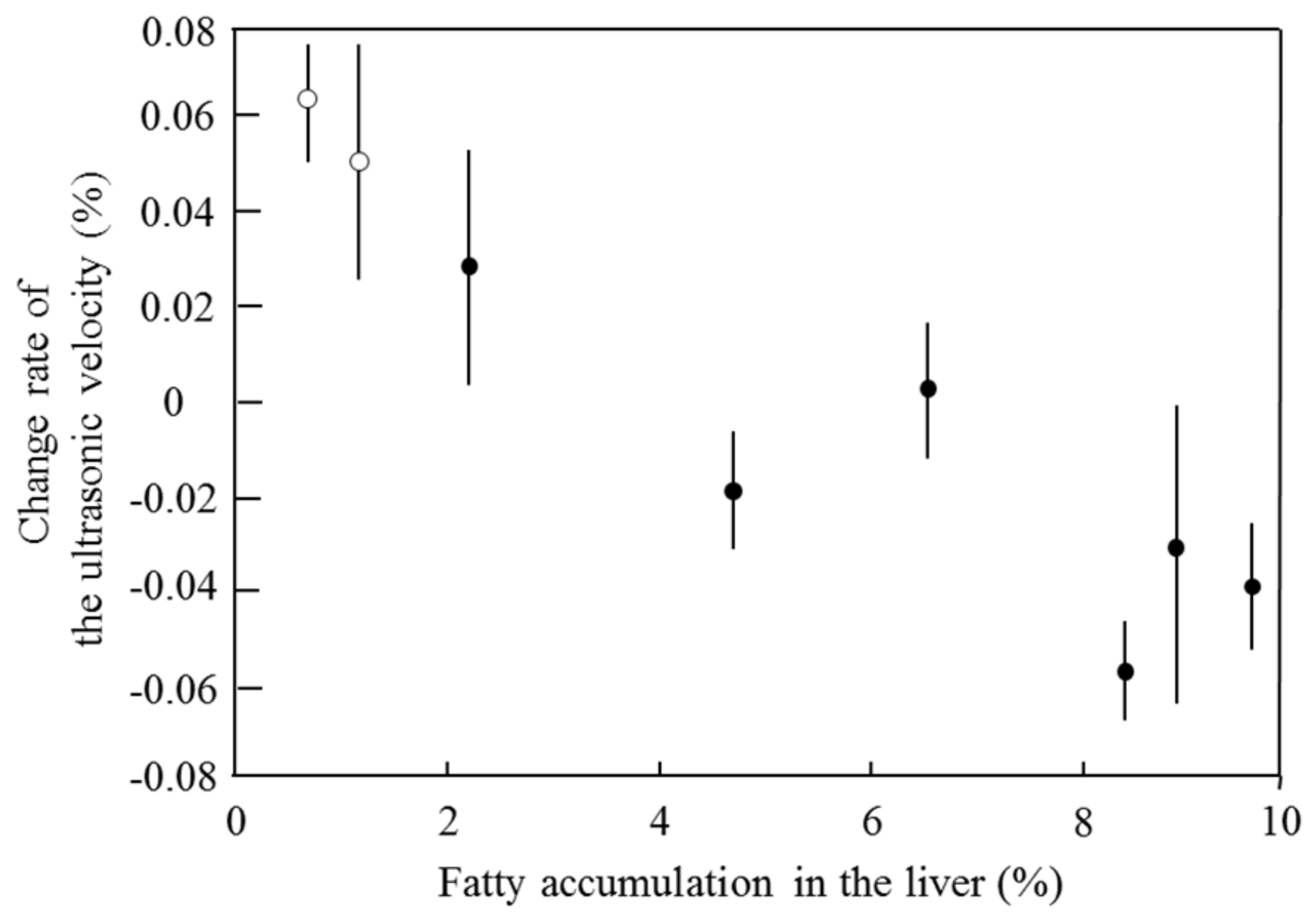

